\title{
Removal of a retained guide wire in a critically ill patient with coagulopathy and cardiac instability
}

\author{
SK Hewage ${ }^{I^{*}, \boldsymbol{R} \text { Amarasena }}{ }^{2}$, \\ Registrar Anaesthsiology ${ }^{l}$, Consultant Anaesthetist ${ }^{2}$, National Hospital of Sri Lanka, Sri Lanka.
}

Loss of a guide wire in the venous system is a dreaded complication following central venous catheter insertion. Removal can be even more challenging in a patient with haemodynamic instability, acute kidney injury and thrombocytopenia. We report a case of successful removal of a retained guide wire lodged in the superior vena cava using correctly timed interventional radiology.

Keywords: Retained guide wire; interventional radiology; leptospirosis

\section{Introduction}

Central venous catheters are used as a route for vasopressor therapy and for parental administration. Depending on the requirement cannulation can be performed at different sites. One of the extremely rare complications is intra vascular loss of the guide wire, which is usually recognized immediately. We elucidate a case of retained guide wire in the venous system following attempted femoral venous cannulation, which was successfully removed by a minimally invasive method.

\section{Case Report}

A 27-year-old gentleman presented to the medical ward in a rural hospital with complaints of generalized abdominal pain, diarrhoea and fever for 5 days' duration. The patient was found to be dehydrated with jaundice and was in shock on admission. His investigations revealed neutrophil leukocytosis, low platelet count $\left(85,000 / \mathrm{mm}^{3}\right)$, elevated liver enzymes, abnormal renal functions and normal coagulation profile. A tentative diagnosis of leptospirosis with myocarditis was made.

*Correspondence: S Hewage

E mail: sachhewage@yahoo.com

Received: 09/05/2017

Accepted: 05/06/2017

DOI: 10.4038 /slja.v25i2.8234

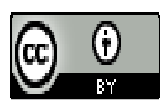

Initial fluid resuscitation and administration of broad spectrum antibiotics did not show signs of improvement. He was immediately admitted to the ICU. Central venous cannulation was attempted in the ICU in view of starting a vasopressor.

The cannula was inserted to the right femoral vein by a medical officer using Seldinger technique. The landmark technique was used since ultrasound scan was not available. Internal jugular vein was not considered for cannulation since the patient refused to stay in head low position. During the procedure, the guide wire had accidentally dislodged and was not accessible to pull out. Subsequent insertion of a right internal jugular central venous line was done by a consultant anaesthetist.

Following stabilization a chest X-ray was taken (Figure 1), which showed that the guidewire tip had migrated via inferior vena cava and was lodging in the superior vena cava.

The patient was transferred to a tertiary care centre for vascular surgical opinion. Surgical removal of the guide wire was delayed initially due to haemodynamic instability and thrombocytopaenia. Patient was admitted to surgical ICU and was closely monitored and leptospirosis management continued. The following day patient developed multiple atrial ectopics in the ECG. Cardiology opinion was sought, the ectopics were thought to be due to the guide wire and not due to myocardial dysfunction. 


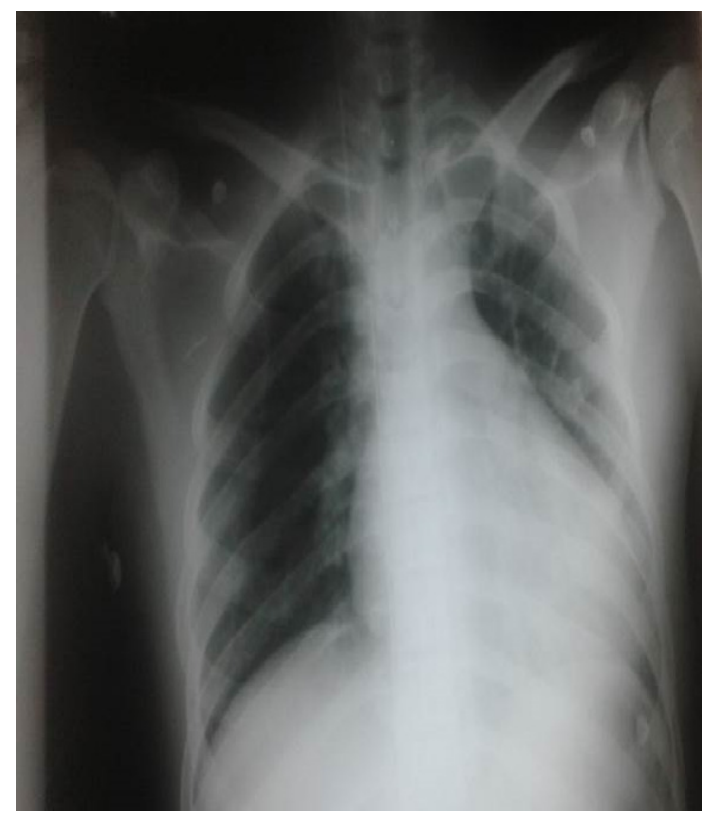

Figure 1

Eight days later patient was haemodynamically stable, vasopressors were tailed off, acute kidney injury (AKI) had resolved and platelet counts improved up to $100,000 / \mathrm{mm}^{3}$. A multidisciplinary team decision was taken to remove the guide wire under radiology guidance. It was removed using a goose-neck snare via right internal jugular vein catheter. Contrast material was avoided due to recent AKI. He had an uneventful recovery and $48 \mathrm{hrs}$ later he was discharged from hospital.

\section{Discussion}

We report an incident of accidental loss of the guide wire in the venous system during femoral venous cannulation. This is a rare and completely avoidable complication. ${ }^{1}$ The most commonly reported guidewire related complications are

\section{- $\quad$ Cardiac dysrhythmias}

- Cardiac conduction abnormalities

- $\quad$ Perforation of vessels or heart chamber

- $\quad$ Kinking, looping or knotting of guidewire

- Entanglement of previously placed intravascular devices

- $\quad$ Breakage of the distal tip of the guidewire with subsequent embolization

To prevent all the above-mentioned complications, the guide wire should be held at the tip at all times.
The diagnosis is easy and immediate. If this complication occurs, interventional radiology technique is preferred than open surgery. ${ }^{2}$ Use of modern devices help to remove a lost catheter, or a guide wire fragment with minimum invasiveness. Our patient posed a unique challenge since he was initially having thrombocytopenia and AKI. We managed to avoid risk of contrast induced nephropathy, by not using contrast material.

For prevention of such an incident in future certain precautions should be followed.

- To follow a unit protocol for central venous line insertion

- Inspect the guide wire for defects before insertion

- When resistance is met not to insert the guide wire forcefully

- Make sure the tip of the guide wire is firmly held before advancing the catheter

- Always inspect the wire following the procedure Multiple human factors may also predispose to occurrence of this event including overtired staff performing cannulation procedures at late night, inattention to the procedure and lack of experience in the technique. To prevent future occurrence of such events human factors has to be taken into consideration.

\section{Conclusion}

Our patient with a retained guide wire in the venous system complicated by cardiac dysrhythmia due to its irritation posed a unique challenge since he was also suffering from leptospirosis with haemodynamic instability, thrombocytopenia and AKI. He was managed successfully without further complications by multidisciplinary team management by properly timed minimally invasive interventional radiology guided technique.

\section{References}

1. Wolf F, Schernthaner RE, Dirisamer A et al. Endovascular management of lost or misplaced intravascular objects; experience of 12 years. Cardiovasc Intervent Radiol, 2008;31(3):563.8

2. Abuhasna S, Abdullah D. The forgotten guide wire, J clin imaging sci $2011 ; \mathbf{1} ; 40$ https://doi.org/10.4103/2156-7514.83397 PMid:21966636 PMCid:PMC3177414 